\title{
Wearable Biomonitoring Monopole Antennas Using Inkjet Printed Electromagnetic Band Gap Structures
}

\author{
Sangkil Kim*, Manos M Tentzeris ${ }^{* *}$ \\ School of Electrical and Computer Engineering \\ Georgia Institute of Technology \\ Atlanta, GA 30332-250, USA \\ ksangki13@gatech.edu*, etentze@ece.gatech.edu ${ }^{* *}$
}

\author{
Symeon Nikolaou \\ Electrical Engineering Department, \\ Frederick University \\ Cyprus \\ s.nikolaou@frederick.ac.cy
}

\begin{abstract}
In this paper, a monopole antenna with a novel nanotechnology-enabled inkjet printed electromagnetic band gap structure (EBG) is introduced for wearable biomonitoring applications with minimal "on-body" performance degradation. The proposed antenna is very thin and flexible since it can be easily fabricated with direct inkjet-printing on commercially available plastic or photo paper, potentially enabling wearable wireless sensing modules with improved communication range.
\end{abstract}

Keywords-wearable antenna, body area networks (BANs), electromagnetic band gap structure (EBGs), artificial magnetic conductor (AMC), nanotechnology, biomonitoring, inkjet printing

\section{INTRODUCTION}

IEEE 802.15 standard has been established to standardize nodes/modules for Body Area Networks (BANs) and Personal Area Networks (PANs), which are getting more and more important due to the ever increasing plethora of demands for wearable radio applications, such as tracking, mobile computing, health monitoring, and public safety. The major challenge for the practical implementation of such wearable radio systems is the degrading effect of the human body, [1][2] as it is effectively a very lossy dispersive material with high dielectric constant absorbing a large amount of the radiated electromagnetic power. Therefore, it is very common for the gain of the antennas to decrease when placed closed to the human body which causes impedance mismatch for the freespace optimized antennas, as well as significant efficiency deterioration, hence, leading to drastic reduction of the communication range of the radio system as well. In addition, the body-absorbed electromagnetic power may cause adverse biological effects. To overcome these challenges, a monopole antenna backed by electromagnetic band gap structure (EBGs) - printed utilizing nanosphere-based conductive inks - is proposed in this paper. The combination of EBGs and ground plane insulate the radio system from the human body effects alleviating the common on-body antenna performance degradation. The proposed antenna can be easily integrated with commercial wireless sensors enabling the development of large scale wireless sensor networks (WSN) with significantly enhanced communication range.

The current paper is structured as follows: First, the inkjet printing technique is discussed in depth. Next, the EBG-backed monopole antenna is presented. Finally, the proposed antenna is connected with a commercially available temperature sensor module, and its communication range improvement is demonstrated.

\section{INKJET PRINTING ON PAPER SUBSTRATE}

As a proof of concept of the proposed configuration, a prototype including an EBG structure is inkjet-printed on the commercially available photo paper using conductive silvernanoparticle based ink. The inkjet printing technology has a lot of advantages. It is extremely low cost, environmentally friendly and enabling fast fabrication. The paper material used as a substrate for this antenna design is organic, biocompatible and renewable material. In addition, inkjet printing technique is different from etching which is a subtractive method by removing unwanted metal from the substrate surface. It jets the ink drops from the nozzle to the desired position. Therefore, it creates no waste, and it results in an economical fabrication process [3].

For the reported fabrication, the DMP2800 inkjet printer is used [4]. For printing, the Dimatix 10pL cartridge (DMC11610) was used, and it was kept at a distance of $800 \mu \mathrm{m}$ from the surface of the paper on the printing plate of the DMP-2800 printer. The printer head was adjusted to achieve a print resolution of 1270 dpi. Cabot conductive ink CCI-300 [5] was jetted at a temperature of $36^{\circ} \mathrm{C}$, while the paper substrate was maintained at $50^{\circ} \mathrm{C}$. The printed pattern was sintered in a thermal oven for 2 hours at $130^{\circ} \mathrm{C}$. After sintering, the pattern has consistent DC conductivity in the range $9 \times 10^{6} \mathrm{~S} / \mathrm{m} \sim$ $1.1 \times 10^{7} \mathrm{~S} / \mathrm{m}$ with roughness of $1 \mu \mathrm{m}[6]$.

Using paper as RF substrate for applications such as RFIDs or antenna-sensor integration has a lot of advantages since large scale production can be feasible when it is combined with inkjet printing technology. The overall cost of the large scale production can be significantly reduced since paper is one of the cheapest commercially available materials. In an additional advantage important for wearable electronics, paper can even become hydrophobic when its surface is coated by proper water resist chemical. The properties of the used paper substrate were studied and characterized using the microstrip ring resonators method [7]. The reported dielectric constant $\left(\varepsilon_{\mathrm{r}}\right)$ of a $0.23 \mathrm{~mm}$ thick paper substrate is 3.2 at $2.4 \sim 2.5 \mathrm{GHz}$, and the loss tangent $(\tan \delta)$ is 0.07 throughout the

This research was supported by Semiconductor Research Corporation/Interconnect Focus Center (SRC/IFC) and NSF ECS 


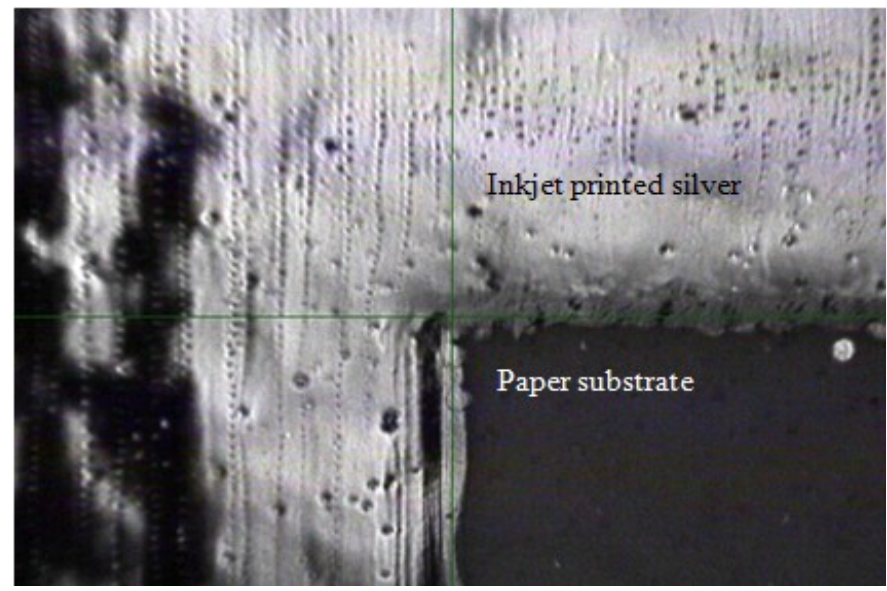

Figure 1. The surface of printed pattern after sintering at $130{ }^{\circ} \mathrm{C}$ for 2 hours

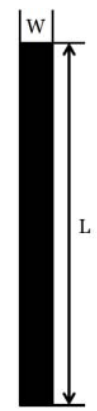

(a)

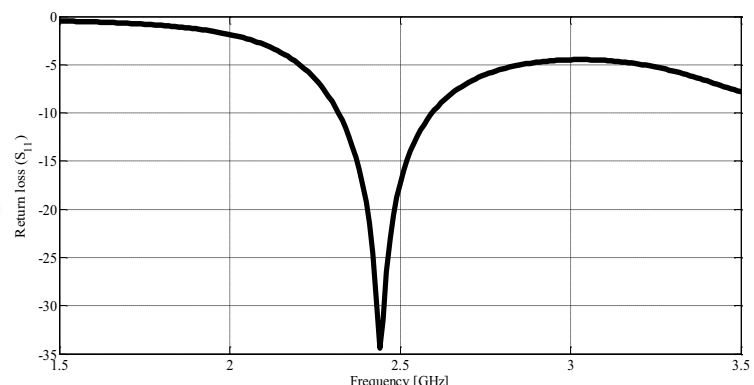

(b)
Figure 2. (a) Schematic of a microstrip monopole antenna ( $\mathrm{W}=1.3 \mathrm{~mm}, \mathrm{~L}=$ $37.2 \mathrm{~mm})(\mathrm{b})$ return loss $\left(\mathrm{S}_{11}\right)$ of the antenna

aforementioned frequency band [8]. Figure 1 shows magnified view of inkjet printed pattern on paper substrate. The printed pattern was sintered at $130{ }^{\circ} \mathrm{C}$ for 2 hours in order for the ink solvent to dry and increase the conductivity of the printed pattern.

\section{ANTENNA DESIGN AND WEARABLE SENSOR NODE PROTOTYPE}

\section{A. Antenna Design}

To demonstrate the enhanced performance of the EBGbacked wearable antennas for practical biomonitoring wireless sensor nodes, a simple microstrip fed monopole antenna was designed to cover the whole IEEE 802.15 frequency band of $2.4 \sim 2.5 \mathrm{GHz}$ backed with a combined inkjet printed EBG layer and an uniform metallic layer acting like an artificial magnetic conductor (AMC) reflector at the designed frequency. The designed monopole antenna is shown in Figure 3. The width of the monopole antenna is $1.3 \mathrm{~mm}$ and its length is $37.2 \mathrm{~mm}$. The frequency response of the antenna shows good matching $\left(\mathrm{S}_{11}<-10 \mathrm{~dB}\right)$ the desired frequency range of $2.4 \sim$ 2.5GHz. The AMC reflector was designed and optimized using the reflection phase characterization method [9][10] in order to increase the gain of the antenna and block the radiated electromagnetic waves in the direction of the human body. Figure 3 (a) shows the picture of the fabricated EBG structures

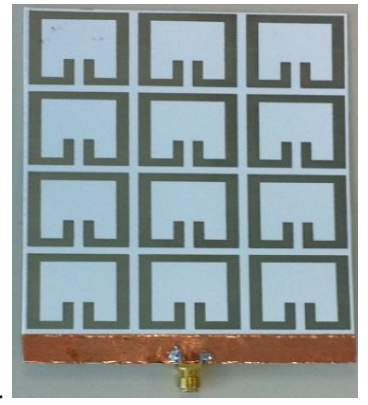

(a)

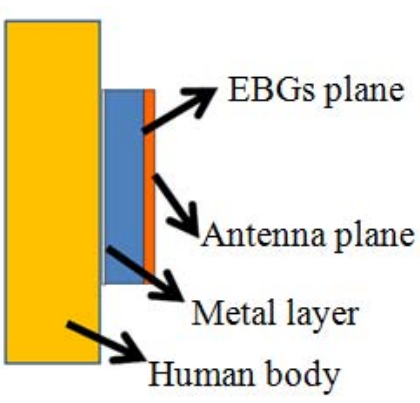

(b)

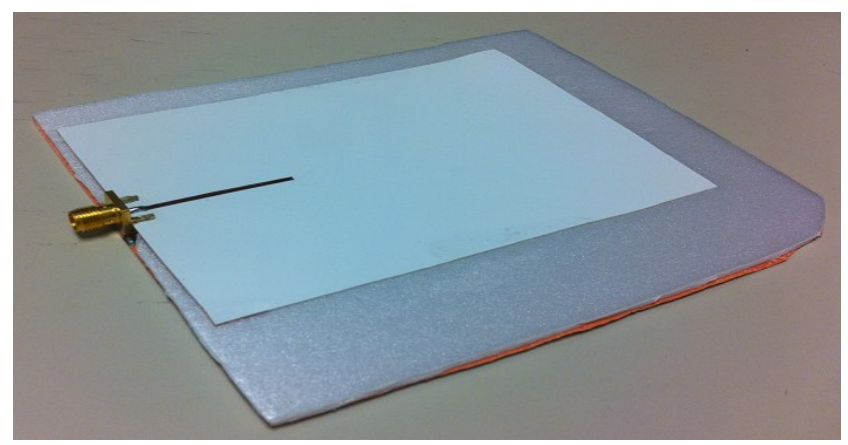

(c)

Figure 3. (a) Inkjet printed EBGs (b) On-body implementation (c) Fabricated antenna

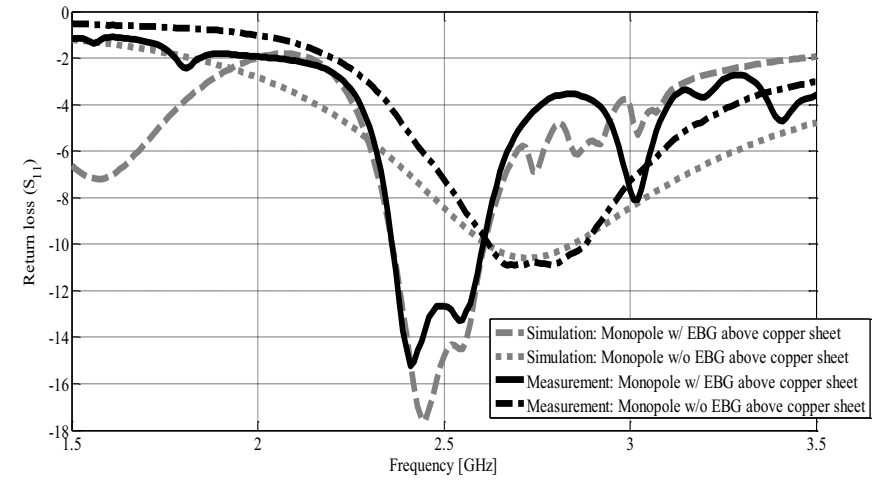

Figure 4. Return loss $\left(\mathrm{S}_{11}\right)$ measurement

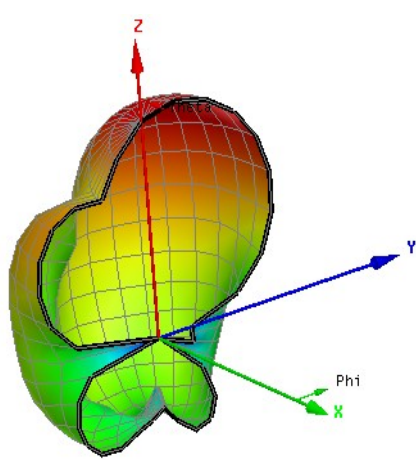

(a) E-plane

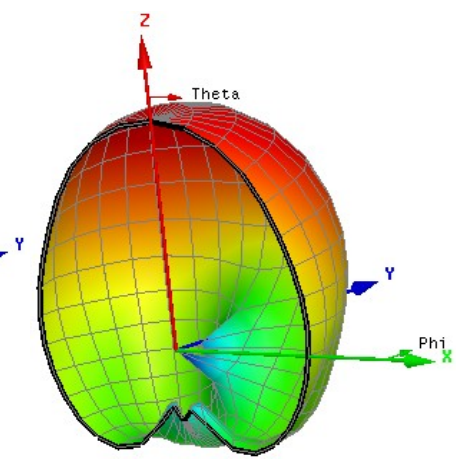

(b) H-plane
Figure 5. Radiation pattern on-body (a) E-plane (b) H-plane 


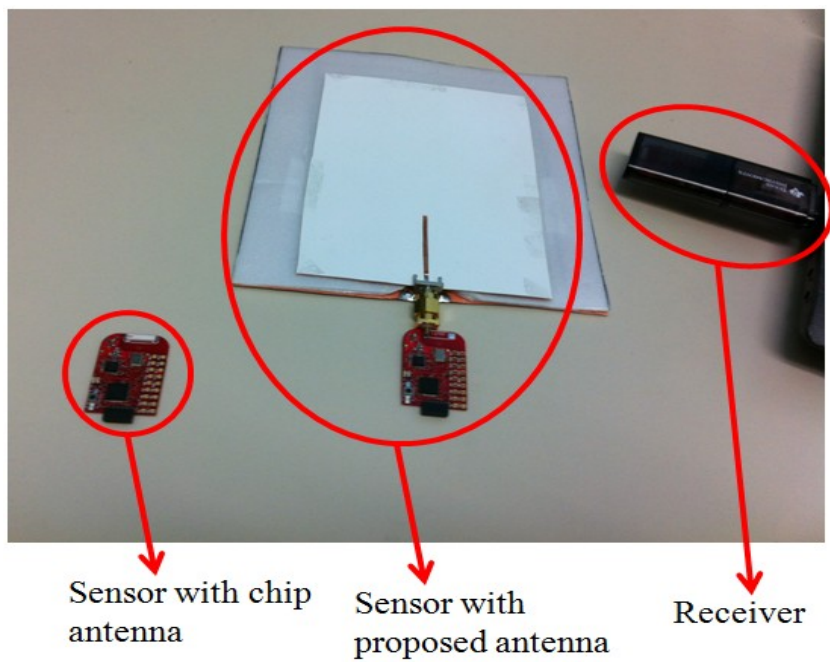

(a)
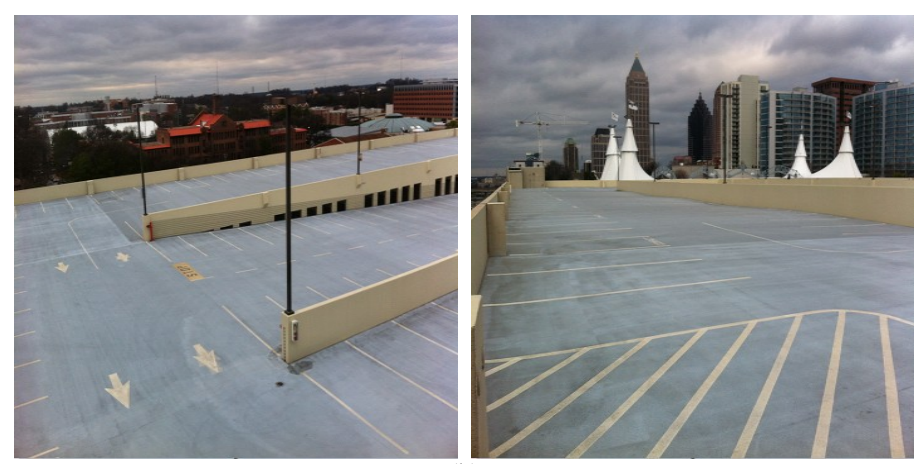

(b)
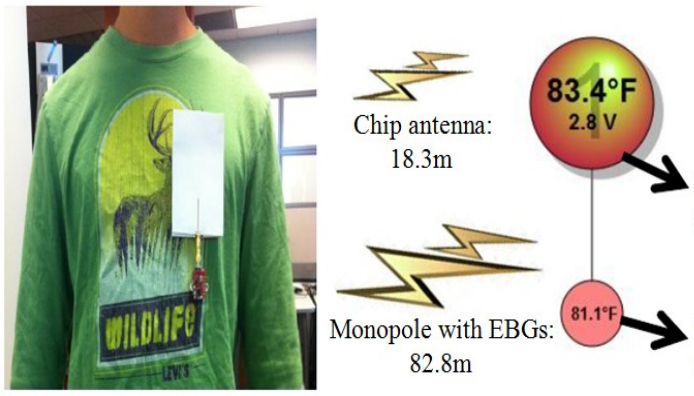

Temperature around transmitter

(c)

Figure 6. (a) Communication range measurement components (Fabricated sensors and receiver) (b) Measurement environment: Parking deck in the urban area (c) Measurement results

and the schematic in 3 (b) shows a side view model of the schematic on top of human body. The board dimensions of the fabricated antenna are $87 \mathrm{~mm} \times 127 \mathrm{~mm} \times 3 \mathrm{~mm}$ and the top view of the fabricated prototype is shown in Figure 3(c). A Styrofoam spacer with the dielectric constant close to air was inserted between the bottom copper metal layer and the EBGs to provide the mechanical support for the implemented AMC.

\section{B. Antenna Simulation and Measurement Results}

Figure 4 shows the reported return loss $\left(\mathrm{S}_{11}\right)$ for the designed monopole when EBG is included (w/EBG) in comparison with a monopole without the printed EBG layer (w/o EBG). It is evident from the plot that simulations and measurements are in good agreement, and also, that EBG layer is necessary in order to achieve the antennas' adequate performance in the presence of the metal layer which is necessary to ensure the shielding of the human body from electromagnetic radiation. Figure 5 shows the simulated $3 \mathrm{D}$ radiation patterns at $\mathrm{f}=2.45 \mathrm{GHz}$. The dark line boundaries of the surface show E- and H- plane distribution of E-co. The existence of the AMC results into a very high front-to-back ratio of $24.3 \mathrm{~dB}$.

\section{Wearable Sensor Node}

The fabricated antenna was integrated with a commercially available wireless temperature sensor in order to demonstrate its significantly improved communication range. For this test, Texas Instrument's eZ430-RF2500 development tool [11] was modified to setup the range measurement. Figure 6 shows the experimental setup along with the measured values. Figure 6(a) shows the communication range measurement components. A transmitting module, eZ430-RF2500T target board, is modified to integrate the antenna to the sensor module. The original chip antenna (Texas Instrument's AN048) on the sensor module (eZ430-RF2500T target board) is replaced by a female SMA connector, and the proposed antenna is mounted. The original chip antenna and the proposed monopole antenna backed with AMC reflector were connected with the sensor module in order to compare communication range as shown in Figure 6(a). The receiver has a USB debugging interface which can be used as a stand-alone system with or without external sensors, or may be incorporated into an existing design. For the receiver the original chip antenna was used. The range measurement took place on the top of the parking deck in the urban area as shown in Figure 6(b). The area of the parking deck is about $40 \mathrm{~m} \mathrm{x}$ $90 \mathrm{~m}$, and it is surrounded by $1 \mathrm{~m}$ tall wall, thus limiting the multipath propagation effect. The receiver was placed on a $20 \mathrm{~cm}$ thick microwave Styrofoam with dielectric constant close to air's $\left(\varepsilon_{\mathrm{r}} \approx 1\right)$. During the measurement the transmitter was attached on the human body as shown in Figure 6(c). The transmitter's distance from ground was about $1.2 \mathrm{~m}$. The receiver was originally set at close distance (about $5 \mathrm{~m}$ ) from the transmitter in order to ensure a good reading. The antennas' alignment was kept in their general maximum directivity direction. Next, the distance from the receiver was gradually increased until the receiver was unable to detect any signal from the transmitting module. The large orange circle which displays the temperature around the transmitter in Figure 6(c) disappears when a receiver lost signal from the transmitter. The distance at which the receiver cannot detect the transmitted signal was marked and the distance between the receiver and the transmitter was measured, using measuring tape. The measured on-body communication range of the sensor module with the chip antenna was $18.3 \mathrm{~m}$, while with the proposed antenna the communication range was increased to $82.8 \mathrm{~m}$, more than four-times of the chip antenna's range.

\section{CONCLUSION}

In this paper, a monopole antenna backed by an EBG/metal layer realization of an artificial magnetic conductor on paper substrate is proposed to alleviate the on-body effects on 
wearable wireless sensors. Preliminary results show that the on-body communication range is improved by approximately a factor of four compared to conventional surface mounted chip antenna.

\section{REFERENCES}

[1] N. H. M. Rais, P. J. Soh, F.Malek, S. Ahmad, N.B.M. Hashim, and P.S Hall, "A Review of Wearable Antenna," Loughborough Antennas \& Propagation Conference, 2009( LAPC 2009), Loughborough, UK, pp225-228, 2009

[2] P. S. Hall, and Y. Hao, "Antennas and Propagation for Body Centric Communications," European Conference on Antennas and Propagation (EuCAP), pp1-7, 2006

[3] V. Lakafosis, A. Rida, R Vyas, Y. Li, S. Nikolaou, M.M. Tentzeris, "Progress Towards the First Wireless Sensor Networks Consisting of Inkjet-Printed, Paper-Based RFID-Enabled Sensor Tags," Proceedings of the IEEE., vol. 98, issue 9, pp. 1601-1609, 2010.

[4] http://www.dimatix.com/

[5] http://www.cabot-corp.com/New-Product-Development/PrintedElectronics/Products

[6] G. Shaker, S. Safavi-Naeini, N. Sangary, M.M. Tentzeris, "Inkjet Printing of Ultrawideband (UWB) Antennas on Paper-Based Substrates," IEEE Antennas and Wireless Propagation Letters, vol. 10, pp. 111-114, 2011.

[7] M. D. Janezic and J. Baker-Jarvis, "Full-wave analysis of a splitcylinder resonator for nondestructive permittivity measurements," IEEE Trans. Microw. Theory Tech., vol. 47, no. 10, pp.2014-2020, Oct. 1999.

[8] Y. Li, A. Rida, R. Vyas, M.M. Tentzeris, "RFID Tag and RF Structures on a Paper Substrate Using Inkjet-Printing Technology," IEEE Trans. Microw. Theory Tech., vol. 55, issue 12, pp. 2894-2901, 2007.

[9] D. Sievenpiper, L. Zhang, R. F. J. Broas, N. G. Alexopoulos, and E. Yablonovich, "High-impedance electromagnetic surfaces with a forbidden frequency band," IEEE Trans. Microw. Theory Tech., vol. 47, pp. 2059-2074, 1999.

[10] F. Yang, Y. Rahmat-Samii, "Reflection phase characterization of the EBG ground plane for low profile wire antenna applications," IEEE Trans. Antennas Propagat., vol. 51, no. 10, pp.2691-2703, 2003.

[11] http://www.ti.com/tool/ez430-rf2500 